\title{
Observation of dia- and paramagnetic domains in beryllium and white tin by muon spin rotation spectroscopy
}

\author{
G. Solt and C. Baines \\ Paul Scherrer Institut, CH-5232 Villigen PSI, Switzerland \\ V. S. Egorov \\ Russian Research Center “Kurchatov Institute," Moscow 123182, Russia \\ D. Herlach and U. Zimmermann \\ Paul Scherrer Institut, CH-5232 Villigen PSI, Switzerland
}

Dia- and paramagnetic (Condon) domains in simple metals, produced at low temperatures and in high magnetic fields via the cooperative effect of electrons in Landau orbitals, are observable by the muon spin rotation $(\mu \mathrm{SR})$ technique. For beryllium, domains were seen for $T<3.5 \mathrm{~K}$ in fields 1 $<H<3 \mathrm{~T}$ through a well resolved splitting of the muon precession frequency, the split lines reappearing in each de Haas-van Alphen (dHvA) period as the applied field $H$ varies. The beat in the dHvA oscillations allowed varying of the amplitude $\chi_{0}$ of the differential susceptibility, the key parameter determining domain properties. The data points in the $(B, T)$ plane show that the standard formula for $\chi_{0}(B, T)$ fails to describe the phase diagram, a consequence of the nearly cylindrical Fermi surface of Be. For white tin, preliminary data indicate the presence of domains by the oscillatory behavior of the $\mu \mathrm{SR}$ linewidth at $T=0.1 \mathrm{~K}$ in fields of $H \approx 1 \mathrm{~T}$, arising from electrons in the pocket of the Fermi surface in the sixth zone. (C) 2000 American Institute of Physics. [S0021-8979(00)67708-3]

\section{INTRODUCTION}

A phase with magnetic domains in nonmagnetic metals at low temperatures, as a consequence of large amplitude de Haas-van Alphen (dHvA) oscillations, was predicted by Condon $^{1}$ on the basis of theoretical arguments as well as of the anomalous waveforms of various oscillating properties of beryllium metal. Direct evidence for domains could subsequently be obtained for silver ${ }^{2}$ at very high fields $(H$ $=9 \mathrm{~T}$ ), but quadrupole broadening and long thermalization times precluded similar NMR studies for other metals, in particular for beryllium.

Exotic at first sight, the formation of Condon domains is however a general property of a system of magnetically interacting electrons in Landau orbitals, as the argument for a perfect single crystal metal specimen shows. ${ }^{3}$ In this ideal case the amplitude $\chi_{0}$ of the oscillatory dHvA susceptibility $\chi=\partial M / \partial B \approx \chi_{0} \cos \{2 \pi B / \Delta H\}$ becomes arbitrarily large as the field $B$ decreases; $\chi_{0}(B, T) \propto B^{-3 / 2}$ for $B \rightarrow 0$, provided the temperature remains low, $k_{B} T / \beta B<1\left(\beta=e \hbar / m^{*} c, m^{*}\right.$ is the cyclotron mass and $\Delta H$ is the dHvA period) ${ }^{3,4}$ Yet, thermodynamic stability ${ }^{4}$ requires $\partial H / \partial B=1-4 \pi \chi(B)>0$, and this is fulfilled only as long as the amplitude $\chi_{0}$ is not "too" large,

$$
\chi_{0}=(\partial M / \partial B)_{\max }<1 / 4 \pi .
$$

For $\chi_{0}>1 / 4 \pi$ the induction $B$ in the sample will have discontinuities, "leaping over" forbidden sections $\left(B_{1}, B_{2}\right)$ in each dHvA cycle where $4 \pi \chi(B)$ would be larger than unity. ${ }^{1}$ For a thin plate oriented normal to the applied field $\mathbf{H}$, however, flux conservation $\mathbf{B}=\mathbf{H}$ requires, for $B_{1}<H$ $<B_{2}$, the induction lying precisely in this forbidden interval. Condon showed ${ }^{1}$ that for these $H$-values the homogeneus state of the specimen breaks up into domains, magnetized alternatingly parallel and antiparallel to $\mathbf{H}$. As $H$ varies in the forbidden interval, the domain inductions stay constant at the two thermodynamically stable values $B=B_{1}<H$ and $B$ $=B_{2}>H$ (in the dia- and paramagnetic domains, respectively), while the volumes of the domains vary with $H$ to ensure flux conservation by "mixing up" an average induction $\bar{B}=H$. The discontinuous $B(H)$ function and the resulting Condon domains are macroscopic manifestations of a cooperative quantum effect, in a situation when the magnetization $\mathbf{M}$ generated by the electrons in Landau orbitals is decisive in determining these quantum states themselves. ${ }^{5}$ Therein, and in the nature of the related periodic phase transitions lies their theoretical interest.

Muon spin rotation $(\mu \mathrm{SR})$ uses $\mu^{+}$particles implanted in the sample which, via their precession frequencies, provide direct information on the magnetic field distribution in the bulk of the specimen. ${ }^{6}$ A particular advantage of $\mu \mathrm{SR}$ is the absence of both skin effect and quadrupolar broadening $\left(I_{\mu}=1 / 2\right)$, major difficulties for NMR in the present case. The domains could be investigated in Be via monitoring the line splitting in the $\mu \mathrm{SR}$ frequency spectrum, while in Sn the splitting was too small to be resolved; it appears instead as a periodic line broadening. Part of the results concerning data on Be were recently published. ${ }^{7}$

\section{EXPERIMENT}

The experiments were performed at the low temperature $\mu$ SR facility of the Paul Scherrer Institute, Villigen. The Be crystals $\left(0.9 \times 1 \times 0.18 \mathrm{~cm}^{3}\right.$ and $\left.0.9 \times 1.4 \times 0.09 \mathrm{~cm}^{3}\right)$ were cut from a single crystal perpendicular to the [001] axis and put normal to both the applied field $\mathbf{H}$ and the incident, polarized 
$\mu^{+}$beam. The penetration length $\approx 0.06 \pm 0.01 \mathrm{~cm}$ at the given momentum $(\approx 28 \mathrm{MeV} / \mathrm{c})$ ensures that the muons stop in the bulk of the samples. Once stopped at an interstitial lattice site (at time $t=0$ ), the $\mu^{+}$spin starts precessing about the local field $\mathbf{B}$ and at time $t$ (mean lifetime $\tau=2.2 \mu \mathrm{s}$ ) emits a positron preferentially into the instantaneous direction of its spin. Thereby the phase of precession as a function of time becomes observable by the oscillating positron intensity $P(t)$ at the $e^{+}$detectors mounted in the precession plane around the sample. For a specimen with two kinds of domains this leads to a $\mu \mathrm{SR}$ signal,

$$
P(t)=\sum_{j=1,2} a^{(j)} e^{-\lambda^{(j)}}{ }_{t} \cos \left(\gamma_{\mu} B_{j} t+\phi\right)
$$

at each detector, with a phase $\phi$ corresponding to its position; the "asymmetry" $a^{(j)}$ is proportional to the volume fraction of domains with induction $B_{j}$. The frequencies $\gamma_{\mu} B_{j}$ give directly the internal field, the damping rates $\lambda$ are due to the field spread around $B_{j}\left(\gamma_{\mu}\right.$ is the gyromagnetic ratio $135.54 \mathrm{MHz} / \mathrm{T}$ of the muon).

As to the dHvA oscillations of $\chi(B)$, the cigarlike Fermi surface of beryllium results in two nearly equal frequencies for the above orientation $\left(F_{w}=942.2 \pm 0.3 \mathrm{~T}\right.$ and $F_{h}=970.9$ $\pm 0.5 \mathrm{~T}),{ }^{8}$ causing a beat in $\chi$ containing $\approx 33 \mathrm{dHvA}$ cycles. This beat was helpful in mapping a large area of the phase diagram in the $(B, T)$ plane, by allowing an easy variation of the key parameter $\chi_{0}$ within a short $H$-interval.

The white tin specimen $\left(12 \times 18 \times 0.56 \mathrm{~mm}^{3}\right)$ is a crystal of extremely high purity, ${ }^{9}$ cut normal to the [100] direction and the relevant dHvA frequency arising from electrons in the sixth Brillouin zone is $F_{C 1}=440 \pm 5 \mathrm{~T} .{ }^{10}$ All experiments were performed at temperatures $0.1<T<3.5 \mathrm{~K}$ and in applied fields of $1<H<3 \mathrm{~T}$.

\section{RESULTS AND DISCUSSION}

\section{A. Beryllium}

A clear evidence of domain formation is seen in Fig. 1, showing a downwards field scan for the thicker Be sample around $H=2 \mathrm{~T}$ through about three $\mathrm{dHvA}$ periods (the length of the period is given by the "frequencies" $F_{w}, F_{h}$ as $\left.\Delta H=B^{2} / \bar{F} \approx 42 \mathrm{G}\right)$. In the domain sections the $B(H)$ function is double-valued (the line in the $\mu \mathrm{SR}$ frequency spectrum is split), while between two domain sections the sample is uniformly magnetized. The $B(H)$ relation is similar to that in Ref. 2 obtained by NMR for silver, except for the field range, the higher value of the key parameter $4 \pi \chi_{0}$ and a smaller demagnetization factor $n=0.775$ in our case. By the observed jump $\Delta B \approx 34 \mathrm{G}$ seen in Fig. 1 , the formula, ${ }^{2}$

$$
\pi \Delta B / \Delta H=4 \pi \chi_{0} \sin \{\pi \Delta B / \Delta H\},
$$

gives the relative length of the "forbidden" section as $\Delta B / \Delta H=0.77$, to be compared to $\approx 0.69$ observed in the silver specimen. (The much larger value of $\Delta B \approx 34 \mathrm{G}$ for the jump, three times that in the experiment for $\mathrm{Ag},{ }^{2}$ is due to the "scaling" by the longer dHvA period here.) Slightly above $H \approx 20690$ Oe the sample is uniform and magnetized parallel to $\mathbf{H}$. With decreasing $H$, the induction $B$ becomes suddenly



FIG. 1. $B(H)$ diagram at $T=0.5 \mathrm{~K}$, showing the recurrent doublet splitting near a beat maximum. The plateaus show the inductions in the dia- and paramagnetic domains (lower and upper plateau, respectively), which do not vary with $H$ in a domain section. The theoretical curve is for $4 \pi \chi_{0}=3.64$ (demagnetizing factor $n=0.775$ ).

two-valued, and a lower plateau $\left(B_{1}\right)$ appears corresponding to diamagnetic regions. As $H$ further decreases, the upper (paramagnetic) and lower (diamagnetic) plateaus show stable values for domain inductions, while the volume fraction of paramagnetic regions decreases with $H$, until at $H$ $\approx 20665$ Oe the entire sample is a uniform, diamagnetic "domain." On lowering $H$, the state remains uniform and the magnetization in the direction of $\mathbf{H}$ increases, the sample becomes again paramagnetic $(\mathbf{M} \| \mathbf{H})$ as the next domain section begins $(H \approx 20645 \mathrm{Oe})$, with the appearance of the two new plateaus $B_{1}^{\prime}, B_{2}^{\prime}$. In the domain sections, the line intensities $a^{(j)}(H)$ showed the expected linear variation of the partial volumes.

Moving along the beat cycle from the antinode $\chi_{0, \max }$ to the node $\chi_{0, \text { min }} \approx \chi_{0, \text { max }} / 3$, the picture (Fig. 2) changes; the jump $\Delta B$ is much smaller and the domain state is limited to a narrow section $n \Delta B$ within the dHvA cycle. The validity

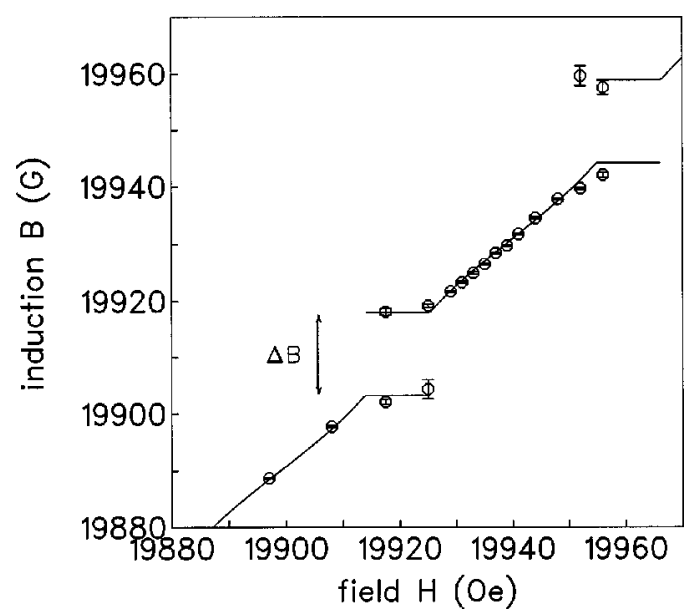

FIG. 2. $B(H)$ diagram at $T=0.5 \mathrm{~K}$ near a beat minimum of $\chi_{0}$. The domain sections are narrower, $\Delta B$ is smaller than in Fig. 1, and the curve is for the fitted value $4 \pi \chi_{0}=1.25$. 


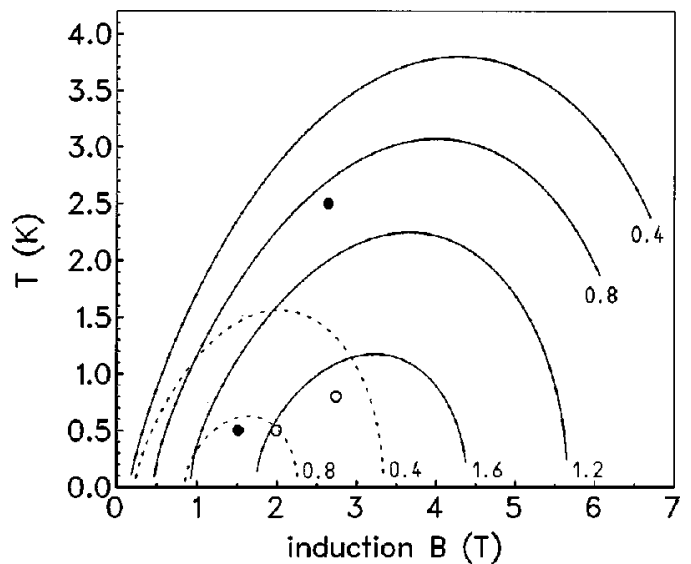

FIG. 3. Calculated phase diagrams for Be, $\mathbf{H} \| c$-axis. The regions below the envelope curves contain the domain regions (the solid and dashed lines correspond to beat maxima and minima, see text). For $T_{D} \geqslant 0.87 \mathrm{~K}$ no domains should appear at the beat nodes, and for $T_{D}>1.78 \mathrm{~K}$ not even at the beat maxima. Yet, the data for two beat antinodes $(\bullet)$ and beat nodes $(\bigcirc)$ do show domain phases, though $T_{D} \approx 2.6 \mathrm{~K}$ for the specimen.

of Eq. (5) is confirmed by the fitted values of $\chi_{0}$ at the maxima and minima, giving $\chi_{0, \min } / \chi_{0, \max }=1.25 / 3.64=0.34$, near the expected $1 / 3$. Similarly, increasing $T$ for $B$ fixed within a domain section, the discontinuity $\Delta B$ was seen to decrease and disappear at $T_{0}$ where the phase boundary $4 \pi \chi_{0}\left(B, T_{0}\right)=1$ is crossed.

The critical parameter $\chi_{0}(B, T)$ and thereby the boundaries for domain formation in the $(B, T)$ plane (below the curves $\left.4 \pi \chi_{0}=1\right)$ can be calculated from the Fermi surface parameters for any given crystal perfection (characterized by the Dingle temperature $T_{D}$ ), normally by using the standard "Lifshitz-Kosevich" (LK) formula. ${ }^{4}$ The calculated phase diagrams are drawn in Fig. 3 for various values of $T_{D}$. For simplicity, only the curves $4 \pi \chi_{0, \max }=1$ and $4 \pi \chi_{0, \min }=1$ are shown, the line $4 \pi \chi_{0}=1$ oscillates between these envelopes. ${ }^{7}$ The data points for our sample with $T_{D}=2.6 \mathrm{~K}$ indicate, however, that the domain regions are far more extended on the phase diagram than standard theory predicts. The explanation lies in the quasi-2D shape of the Fermi surface, and the approximations implied in deriving the threedimensional LK formula are inadequate for dealing with the nearly cylindrical "cigars" in this case. In fact, a much simplified quasi-2D treatment ${ }^{11}$ gives, in order of magnitude, better "average" values for the phase envelopes in this case, though leaving aside, in turn, important features due to the presence of two dHvA frequencies, an inherently threedimensional property.

\section{B. White tin}

The Fermi surface of white tin has a cube-formed "pocket" in the sixth zone, and the slow variation of the cross section $A$ along the [100] direction indicates also here a large dHvA amplitude $\left[\chi_{0} \propto\left(\left|A^{\prime \prime}\right|\right)^{-1 / 2}\right.$ (Ref. 4)] favorable for domain formation. The observed damping rate (line broadening) near $H=1 \mathrm{~T}$, in Fig. 4, shows indeed oscillations with the dHvA period $H^{2} / F_{C 1}=23 \mathrm{G}$ which must be due to a line

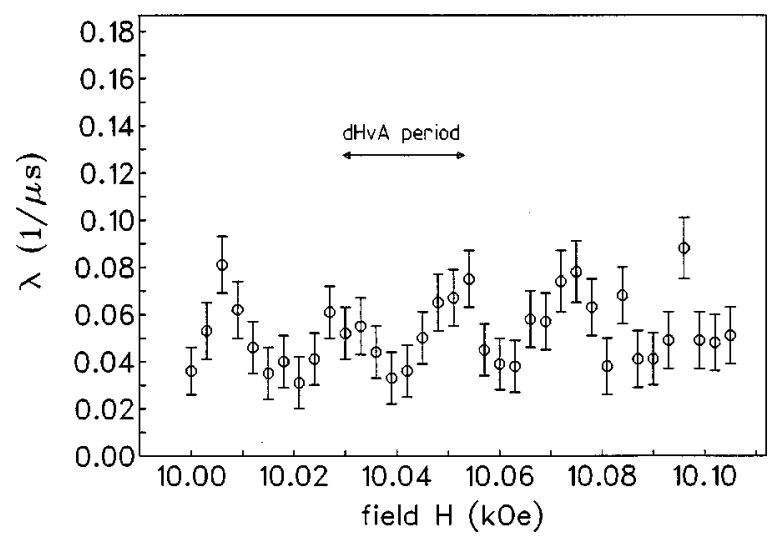

FIG. 4. Damping rate (linewidth) for white tin, at $T=0.1 \mathrm{~K},[100] \| \mathbf{H}$. The oscillations, having the dHvA period, indicate line splitting due to domain sections in each dHvA cycle $\left(\Delta H=B^{2} / F_{C 1}, F_{C 1}=440 \pm 5 \mathrm{~T}\right)$.

splitting, though not sufficiently large to be resolved as in Fig. 1. To resolve the doublets, further experiments with improved resolution and at higher fields $\left(\Delta H \propto H^{2}\right)$ are under progress.

\section{CONCLUSIONS}

Condon domains and the variation of the domain parameters, in particular the discontinuity $\Delta B$ and the length of the domain section in the dHvA cycle, were observed for a wide range of the key parameter $\chi_{0}(B, T)$ in beryllium. Once fitting $\chi_{0}$ to the data, the theory describes the $B(H)$ relation correctly both in the domain sections and in the uniform state. However, the $a b$ initio calculation of $\chi_{0}(B, T)$ by the LK formula fails to reproduce the observed phase diagrams, as a consequence of the quasi-2D feature of the energy spectrum at the Fermi surface. Since the actual phase boundaries reflect both this quasi-two-dimensionality and also the hipsand-waist structure of the electron "cigars," to remedy this situation a more sophisticated calculation is needed, taking into account in a more complete way the particular shape of the Be Fermi surface. The results for white tin confirm that Condon domain formation is a general property of sufficiently pure metal crystals. The low curvature along $\mathbf{H}$ at the extremal cross sections of the Fermi surface, as here for Be and $\mathrm{Sn}$, is particularly favorable for domain formation, making the temperature and purity requirements less restrictive.

${ }^{1}$ J. H. Condon, Phys. Rev. 145, 526 (1966).

${ }^{2}$ J. H. Condon and R. E. Walstedt, Phys. Rev. Lett. 21, 612 (1968).

${ }^{3}$ A. B. Pippard, in Electrons at the Fermi Surface, edited by M. Springford (Cambridge University Press, Cambridge, 1980), p. 124.

${ }^{4}$ L. D. Landau and E. M. Lifshitz, Statistical Physics (Pergamon, Oxford, 1980), Part 2.

${ }^{5}$ D. Shoenberg, Magnetic Oscillations in Metals (Cambridge University Press, Cambridge, 1984).

${ }^{6}$ A. Schenck, Muon Spin Rotation Spectroscopy (Hilger, Bristol, 1986).

${ }^{7}$ G. Solt, C. Baines, V. S. Egorov, D. Herlach, and U. Zimmermann, Phys. Rev. B 59, 6834 (1999).

${ }^{8}$ V. S. Egorov, Sov. Phys. Solid State 30, 730 (1988).

${ }^{9} \mathrm{We}$ are indebted to Professor V. F. Gantmakher for providing us with the sample.

${ }^{10}$ M. D. Stafleu and A. R. de Vroomen, Phys. Status Solidi 23, 675 (1967).

${ }^{11}$ A. Gordon, M. A. Itskovsky, I. D. Vagner, and P. Wyder, Phys. Rev. Lett. 81, 2787 (1998). 